\title{
DIFFERENCE SCHEMES FOR PARTIAL DIFFERENTIAL EQUATIONS OF FRACTIONAL ORDER
}

\author{
A.K. BAZZAEV, I.D. TSOPANOV
}

\begin{abstract}
Nowadays, fractional differential equations arise while describing physical systems with such properties as power nonlocality, long-term memory and fractal property. The order of the fractional derivative is determined by the dimension of the fractal. Fractional mathematical calculus in the theory of fractals and physical systems with memory and non-locality becomes as important as classical analysis in continuum mechanics.

In this paper we consider higher order difference schemes of approximation for differential equations with fractional-order derivatives with respect to both spatial and time variables. Using the maximum principle, we obtain apriori estimates and prove the stability and the uniform convergence of difference schemes.
\end{abstract}

Keywords: initial-boundary value problem, fractional differential equations, Caputo fractional derivative, stability, slow diffusion equation, difference scheme, maximum principle, stability, uniform convergence, apriori estimate, heat capacity concentrated at the boundary.

Mathematics Subject Classification: 65M06, 35S99

\section{INTRODUCTION}

Integrals and derivatives of fractional order and fractional integro-differential equations are widely used in modern studies in theoretical physics, mechanics and applied mathematics. A fractional mathematical calculus is a powerful tool for describing physical systems with a memory and nonlocality. Many processes in complex systems possess a nonlocality and a longterm memory. Fractional integral and fractional differential operators allows us to describe some of these characteristics. The employing of the fractional mathematical analysis can be useful for obtaining dynamical models, in which integro-differential operators in time and spatial variables describe a power long-term memory and a spatial nonlocality of complex media and processes [1].

The presence of the fractional derivative in time in an equation is interpreted as a reflection of a special property of the described process, a memory, or, for stochastic processes, a non-Markov property. The fractional derivative in time usually reflects a self-similar inhomogeneity of a structure or of a media, in which the process is going on. Such structures are called fractals. At that, the order of the fractional derivative is determined by the dimension of the fractal. Simple formulae relating the dimension of the fractal $d_{f}$ with the order of the fractional derivative were obtained in work [2]. At present, partial differential equations with fractional derivatives in time and spatial variables are treated as mathematical models of physical processes [3]-[6].

To describe the structure of disordered media and the processes in them, the fractal theory is widely used, see [7]-[11]. Examples of disordered media are porous solids. At that, the fractals can be a porous space, the skeleton of a rock, the surface of the skeleton of a rock, etc. In the

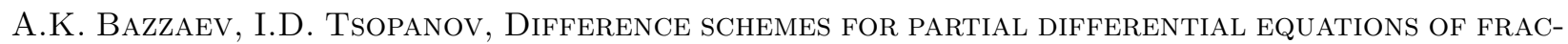
TIONAL ORDER.

(C) Bazzaev A.K., Tsopanov I.D. 2019.

Submitted May 31, 2018. 
case the cracks and solid porous blocks are homogeneous mutually penetrating continuums, the filtration of a homogeneous liquid is usually described by the Barenblatt-Zheltov model, see [12. If the space is a fractal with Hausdorff-Besicovitch dimension $d_{f}$ embedded into a solid media of dimension $d, d>d_{f}, d=2,3$, the motion of an impurity in the flow of a homogeneous liquid is described by a fractional order differential equation, see [13]. A fractional differential equation arises also in studying physical processes of stochastic transfer, see [8].

Boundary value problems for fractional order differential equations arise also in studying of many physical processes [14]-[15], in studying filtration of a liquid in a very porous (fractal) media [16].

A transfer described by operators with fractional derivatives at large distances from the sources gives rise to another behavior of small concentrations in comparison with a classic diffusion. These small concentrations or far tails of distributions at fractional derivative exhibit a power decay that insists on reconsidering the present concepts on a security based on exponential decay rate, see [17, [18. As it was mentioned in [19], a fractional calculus in the fractal theory and systems with memory becomes as important as a classical analysis in the continuum mechanics.

There exist rather many evidences of the fact that a diffusion processes exhibit a nonlinear growth of a mean-square deviation [20]. The violation are observed in many situations, in particular, in motion of particles in plasma [21], a turbulent diffusion of particles [22]. The mathematical models of such processes are described by partial differential equations with fractional derivatives in time and spatial variables [3]- [5]. In work [6], a numerical modelling of an anomalous diffusion in a multi-dimensional domain is made by means of the approximate factorization method. The Dirichlet initial-boundary value problem for a partial differential equations with fractional derivatives in time and spatial variables, there was studied an implicit scheme based on the approximate factorization method and the stability of the scheme was proved for the considered class of the problems.

In work [23, a special semi-discrete scheme based on the Galerkin method was considered as well as a completely discrete scheme based on the Crank-Nicolson method for the Dirichlet initial-boundary value problem for a one-dimensional parabolic equations with fractional Riemann-Liouville derivative of order $\alpha \in(1,2)$ in a spatial variable:

$$
\begin{gathered}
u_{t}-\mathcal{D}_{x}^{\alpha} u=f, \quad x \in D=(0,1), \quad 0<t \leqslant T, \\
u(0, t)=u(1, t)=0, \quad 0<t \leqslant T, \quad u(x, 0)=v, \quad x \in D .
\end{gathered}
$$

There were obtained the estimates for the errors in norms of $L_{2}(D)$ and $H^{\frac{\alpha}{2}}(D)$ for the semidiscrete scheme and in the norm of $L_{2}(D)$ for the completely discrete scheme. The variational formulation of Petrov-Galerkin type for one-dimensional boundary value problems with a fractional Riemann-Liouville derivative of order $\alpha \in\left(\frac{3}{2}, 2\right)$ was considered in work [24]. In work [25], there was considered an equation with fractional in time derivative subject to the Dirichlet condition:

$$
\begin{gathered}
\partial_{0 t}^{\alpha} u-\Delta u=f, \quad x \in \Omega, \quad 0<t \leqslant T, \quad 0<\alpha<1, \\
\left.u\right|_{\Gamma}=0, \quad 0<t \leqslant T, \quad \Omega+\Gamma=\bar{\Omega}, \quad u(x, 0)=v, \quad x \in \Omega .
\end{gathered}
$$

Here a discrete analogue of the fractional derivative in time was obtained; the order of the approximation was $O\left(\tau^{2-\alpha}\right)$. The convergence of the constructed scheme was proven in the norm of $L_{2}(\Omega)$.

In works [26] and [27] there were considered locally-one-dimensional schemes (LOS) for a fractional order diffusion equation in a $p$-dimensional parallelepiped with Dirichlet and Robin conditions, while in [28] the same was made for a fractional heat equation with a concentrated heat capacity. In these works there was proved the convergence of LOS in the uniform metric as $\frac{1}{2}<\alpha \leqslant 1$. In work [29] there were constructed multi-dimensional difference schemes 
for the fractional order diffusion equation and the convergence of the difference schemes was proved for all $\alpha, 0<\alpha \leqslant 1$. Work [30] was devoted to considering locally-one-dimensional difference schemes for a fractional order diffusion equation with variable coefficients in domains of a complicated shape. The stability and the uniform convergence of locally-one-dimensional schemes were proved for the considered problem.

It was shown in [31] that the method of energy inequalities can be employed for obtaining apriori estimates in numerical solving fractional order diffusion equation. In work [32], an algorithm of extrapolation type was proposed for numerical solving fractional order differential equations based on the fact that the sequence of approximated solutions possesses an asymptotic expansion in the step size. Work [33] was devoted to studying existence, uniqueness and stability of solutions to nonlinear differential equations of fractional order in time. In works [34] - 35] there were considered fractional order heat equations with Robin boundary conditions. Work [36] was devoted to a numerical method of second order sharpness for a fractional order diffusion equation. A numerical algorithm proposed in this work, was based on the classical Crank-Nicolson method. The convergence of the proposed method was proved. The maximum principle for a diffusion equation with a fractional in time derivative was established in works by Yu. Luchko [37]-[40]. The results of these works were employed in works [41], [42] to prove the maximum principle for a multi-term fractional differential equation. In work [43] there were constructed difference schemes for partial differential equations with fractional derivatives in time and spatial variables in one- and multi-dimensional cases. In the multi-dimensional case, for the considered problems there were constructed locally-one-dimensional schemes. By means of the maximum principle, apriori estimates were obtained in the uniform metric that implied the convergence of difference schemes.

In work [44] there was proposed a discrete analogue of the fractional Caputo derivative $\partial_{0 t}^{\alpha} u$, $\alpha \in(0,1)$, and it was also shown that functions $u(t) \in C^{2}[0, t]$ satisfy the identity

$$
\partial_{0 t_{j+1}}^{\alpha} u=\Delta_{0 t_{j+1}}^{\alpha} u+O(\tau),
$$

where

$$
\partial_{0 t}^{\alpha} u=\frac{1}{\Gamma(1-\alpha)} \int_{0}^{t} \frac{\dot{u}(x, \eta)}{(t-\eta)^{\alpha}} d \eta
$$

is a fractional Caputo derivative of order $\alpha, 0<\alpha<1, \dot{u}=\partial u / \partial t, \Delta_{0 t_{j+1}}^{\alpha} u$ is a discrete analogue of the fractional Caputo derivative of order $\alpha, \alpha \in(0,1)$,

$$
\Delta_{0 t_{j+1}}^{\alpha} u=\frac{1}{\Gamma(2-\alpha)} \sum_{s=1}^{j}\left(t_{j-s+1}^{1-\alpha}-t_{j-s}^{1-\alpha}\right) u_{t}^{s}, \quad u_{t}^{s}=\frac{u^{s+1}-u^{s}}{\tau} .
$$

Later, in work [45], the following lemma was proved.

Lemma 1. If $u(t) \in C^{3}[0, T]$, then

$$
\partial_{0 t_{j+1}}^{\alpha} u=\Delta_{0 t_{j+1}}^{\alpha} u+O\left(\tau^{2-\alpha}\right), \quad \alpha \in(0,1) .
$$

The present work is devoted to considering difference schemes of higher approximation order for fractional order differential equations.

\section{FRACTIONAL ORDER DIFFUSION EQUATION WITH FRACTIONAL DERIVATIVE IN LOWER ORDER TERMS}

In work [28], implicit schemes and locally-one-dimensional schemes were considered for fractional order diffusion equation subject to Robin condition in a multi-dimensional domain. By means of the maximum principle, stability and uniform convergence of LOS were proved for 
$\frac{1}{2}<\alpha \leqslant 1$, where $\alpha$ was the order of the fractional derivative in time. In work [46], locallyone-dimensional schemes were constructed for the diffusion equation with fractional derivative in spatial variables in lower order terms. The stability and uniform convergence of LOS were proved for $\frac{1}{2}<\alpha \leqslant 1$.

1.1. Formulation of problem. In a rectangle $Q_{T}=\{0 \leqslant x \leqslant \ell, 0<t \leqslant T\}$ we consider a Robin initial-boundary value problem for a fractional order diffusion equation with a fractional derivative $\partial_{0 x}^{\nu} u$ in the spatial variable $x$ of order $\nu, 0<\nu<1$, in the lower order terms:

$$
\begin{aligned}
& \partial_{0 t}^{\alpha} u=\frac{\partial}{\partial x}\left(k(x, t) \frac{\partial u}{\partial x}\right)+r(x, t) \partial_{0 x}^{\beta} u-q(x, t) u+f(x, t), \quad(x, t) \in Q_{T}, \\
& \left\{\begin{array}{c}
k(x, t) \frac{\partial u}{\partial x}=\lambda_{-}(x, t) u-\mu_{-}(x, t), \quad x=0, \quad 0 \leqslant t \leqslant T, \\
-k(x, t) \frac{\partial u}{\partial x}=\lambda_{+}(x, t) u-\mu_{+}(x, t), \quad x=\ell, \quad 0 \leqslant t \leqslant T, \\
u(x, 0)=u_{0}(x), x \in \bar{G},
\end{array}\right.
\end{aligned}
$$

where $0<c_{0} \leqslant k \leqslant c_{1}, r \leqslant 0,|r| \leqslant c_{2}, q>0, \lambda_{ \pm} \geqslant \lambda^{*}>0$,

$$
\begin{array}{ll}
\partial_{0 t}^{\alpha} u=\frac{1}{\Gamma(1-\alpha)} \int_{0}^{t} \frac{\dot{u}(x, \eta)}{(t-\eta)^{\alpha}} d \eta, & 0<\alpha<1, \\
\partial_{0 x}^{\beta} u=\frac{1}{\Gamma(1-\beta)} \int_{0}^{x} \frac{u^{\prime}(\xi, t)}{(x-\xi)^{\beta}} d \xi, & 0<\beta<1,
\end{array}
$$

are the Caputo fractional derivatives of orders $\alpha$ and $\beta$; the latter is in the variable $x$, and $\dot{u}=\partial u / \partial t, u^{\prime}=\partial u / \partial x, c_{0}, c_{1}, c_{2}$ are positive constants, $\bar{Q}_{T}=\{0 \leqslant x \leqslant \ell, 0 \leqslant t \leqslant T\}$.

1.2. Difference scheme. In a closed domain $\bar{Q}_{T}$ we introduce a uniform grid. A spatial grid is chosen to be uniform with the step $h=\ell / N$ :

$$
\bar{\omega}_{h}=\left\{x_{i}=i h: i=0,1, \ldots, N, h=\ell / N\right\} .
$$

By $\omega_{h}$ we denote the set of all interior nodes of the grid $\bar{\omega}_{h}$. On the segment $0 \leqslant t \leqslant T$ we choose the uniform grid

$$
\bar{\omega}_{\tau}^{\prime}=\left\{0, t_{j+1}=(j+1) \tau, \quad j=0,1, \ldots, j_{0}-1\right\} .
$$

By $\omega_{\tau}^{\prime}$ we denote the set of the nodes of the grid $\bar{\omega}_{\tau}^{\prime}$, for which $t>0$.

Similar to [47], for equation (2), we are going to obtain a monotone scheme of second order of approximation in $h$, for which the maximum principle should hold for all $\tau$ and $h$. In order to do this, we consider equation (2) with a perturbed operator

$$
\widetilde{L}=\varkappa \frac{\partial}{\partial x}\left(k(x, t) \frac{\partial u}{\partial x}\right)+r(x, t) \frac{\partial u}{\partial x}-q(x, t) u,
$$

namely,

$$
\partial_{0 t}^{\alpha} u=\widetilde{L} u+f=0
$$

where $\varkappa=\frac{1}{1+R}, R=\frac{0.5 h|r|}{k}$ is a difference Reynolds number.

For a fixed $t=\bar{t}=t_{j+\frac{1}{2}}$, we approximate the operator $\widetilde{L}$ by the difference operator

$$
\widetilde{\Lambda} u=\varkappa\left(a u_{\bar{x}}\right)_{x}+b^{+} a^{(+1)} u_{x}+b^{-} a u_{\bar{x}}-d u,
$$

where

$a=A[k(x+i h, \bar{t})], \quad d=F[q(x+i h, \bar{t})], \quad a^{(+1)}=a(x+i h, \bar{t}), \quad b^{ \pm}=F\left[\widetilde{r}^{ \pm}(x+i h, \bar{t})\right]$, 
$\widetilde{r}^{ \pm}=\frac{r^{ \pm}}{k}, \quad r^{+}=0.5(r+|r|) \geqslant 0, \quad r^{-}=0.5(r-|r|) \leqslant 0$,

$A$ and $F$ are pattern functionals employed for calculating the coefficients $a, d$ and $\varphi$ and ensuring the second order of the approximation. For instance, we can let $b^{ \pm}=\frac{r^{ \pm}}{k}$.

With differential problem (2) - (4), we associate an implicit difference scheme:

$$
\begin{aligned}
& \Delta_{0 t_{j+1}}^{\alpha} y=\bar{\Lambda} y^{j+1}+\Phi^{j+1}, \quad y(x, 0)=u_{0}(x), \\
& \bar{\Lambda} y= \begin{cases}\Lambda y=\left(a y_{\bar{x}}\right)_{x}+\frac{r_{i}}{\Gamma(2-\beta)} \sum_{s=0}^{i}\left(x_{i-s+1}^{1-\beta}-x_{i-s}^{1-\beta}\right) y_{x, s}-d y, & x \in \omega_{h}, \\
\Lambda^{-} y=\frac{\bar{a}^{(1)} y_{x, 0}-\bar{\lambda}_{-} y_{0}}{0.5 h}, & x=0, \\
\Lambda^{+} y=-\frac{\bar{a}^{(N)} y_{\bar{x}, N}+\bar{\lambda}_{+} y_{N}}{0.5 h}+0.5 h r_{N} \Delta_{0 x_{N-1}}^{\beta} y, & x=\ell,\end{cases} \\
& \Phi=\left\{\begin{array}{lll}
\varphi, & x \in \omega_{h}, & \\
\frac{\bar{\mu}_{-}}{0.5 h}, & x=0, & \\
\frac{\bar{\mu}_{+}}{0.5 h}, & x=\ell,
\end{array}\right.
\end{aligned}
$$

where

$$
\begin{aligned}
& \bar{a}^{(1)}=a^{(1)}+\frac{0.5 h^{2-\beta}}{\Gamma(2-\beta)} r_{0}, \quad \bar{a}^{(N)}=a^{(N)}-\frac{0.5 h^{2-\beta}}{\Gamma(2-\beta)} r_{N}, \quad \bar{\lambda}_{-}=\lambda_{-}+0.5 h d^{(0)}, \\
& \bar{\lambda}_{+}=\lambda_{+}+0.5 h d^{(N)}, \quad \bar{\mu}_{-}=\mu_{-}+0.5 h f_{0}, \quad \bar{\mu}_{+}=\mu_{+}+0.5 h f_{N}, \\
& \Delta_{0 t_{j+1}}^{\alpha} y=\frac{1}{\Gamma(2-\alpha)} \sum_{s=0}^{j}\left(t_{j-s+1}^{1-\alpha}-t_{j-s}^{1-\alpha}\right) y_{t}^{s}, \quad y_{t}^{s}=\frac{y^{s+1}-y^{s}}{\tau}, \\
& \Delta_{0 x_{N-1}}^{\beta} y=\frac{1}{\Gamma(2-\beta)} \sum_{s=1}^{N-1}\left(x_{N-s+1}^{1-\beta}-x_{N-s}^{1-\beta}\right) y_{\bar{x}, s}, \quad y_{\bar{x}, s}=\frac{y_{s}-y_{s-1}}{h} .
\end{aligned}
$$

As the solution and the data of problem (2)-(4) is smooth enough, by Lemma 1, difference scheme (6) has the approximation order $O\left(h^{2-\beta}+\tau^{2-\alpha}\right)$.

1.3. Stability and uniform convergence of difference scheme. The following theorem holds.

Theorem 1. Difference scheme (6) is stable with respect to the initial data and the right hand side and the solution of problem (6) satisfies the apriori estimate

$$
\left\|y^{j+1}\right\|_{C} \leqslant\left\|y^{0}\right\|_{C}+\frac{1}{\lambda^{*}} \max _{0<t^{\prime} \leqslant j \tau}\left(\left|\bar{\mu}_{-}\left(x, t^{\prime}\right)\right|+\left|\bar{\mu}_{+}\left(x, t^{\prime}\right)\right|\right)+\Gamma(2-\alpha) \sum_{j^{\prime}=0}^{j} \tau^{\alpha}\left\|\varphi^{j^{\prime}}\right\|_{C},
$$

which implies the convergence of scheme (6) in the uniform metric with the rate $O\left(h^{2-\beta}+\tau^{2-\alpha}\right)$. Proof. We rewrite difference problem (6) as follows:

$$
\begin{aligned}
& \Delta_{0 t_{j+1}}^{\alpha} y_{i}=\left(a y_{\bar{x}}^{j+1}\right)_{x, i}+r_{i} \Delta_{0 x, i}^{\beta} y^{j+1}-d_{i} y_{i}^{j+1}+\varphi_{i}^{j+1}, \quad i=1, \ldots, N-1, \\
& \Delta_{0 t_{j+1}}^{\alpha} y_{0}=\frac{\bar{a}^{(1)} y_{x, 0}-\bar{\lambda}_{-} y_{0}}{0.5 h}+\frac{\bar{\mu}_{-}}{0.5 h}, \quad x=0, \\
& \Delta_{0 t_{j+1}}^{\alpha} y_{N}=-\frac{\bar{a}^{(N)} y_{\bar{x}, N}+\bar{\lambda}_{+} y_{N}}{0.5 h}+0,5 h r_{N} \Delta_{0 x_{N-1}}^{\beta} y+\frac{\bar{\mu}_{+}}{0.5 h}, \quad x=\ell,
\end{aligned}
$$




$$
y(x, 0)=u_{0}(x), \quad x \in \bar{\omega}_{h} .
$$

We obtain apriori estimate for problem (8)-(11) by means of the maximum principle proved in [48] for a grid equation of general form:

$$
A(P)=\sum_{Q \in \mathbb{I}^{\prime}(P)} B(P, Q) y(Q)+F(P)
$$

where

$$
\begin{aligned}
& A(P)>0, \quad B(P, Q)>0, \\
& D(P)=A(P)-\sum_{Q \in \mathbb{I}^{\prime}(P)} B(P, Q) y(Q) \geqslant 0,
\end{aligned}
$$

$P, Q$ are the nodes of the grid $\bar{\omega}_{h}, \mathbb{I I}^{\prime}(P)$ is a vicinity of a node $P$ not containing the node $P$ itself.

By $P\left(x, t^{\prime}\right), x \in \omega_{h}, t \in \omega_{\tau}^{\prime}$ we denote a note of $(p+1)$-dimensional grid $\Omega=\omega_{h} \times \omega_{\tau}$, and by $S$ we denote the boundary of $\Omega$ consisting of the nodes $P(x, 0)$ as $x \in \bar{\omega}_{h}$ and the nodes $P\left(x, t_{j+1}\right)$ as $t_{j+1} \in \omega_{\tau}^{\prime}$ and $x \in \gamma_{h}, j=0,1, \ldots, j_{0}$.

We represent the solution of problem (8) - 111) as the sum

$$
y=\stackrel{\star}{y}+\stackrel{\circ}{y}
$$

where $\stackrel{*}{y}$ is the solution of homogeneous equations (8) with inhomogeneous boundary conditions (11) and homogeneous initial conditions (11):

$$
\begin{aligned}
& \Delta_{0 t_{j+1}}^{\alpha} \stackrel{\star}{y_{i}}=\left(a \stackrel{\star}{y}_{\bar{x}}^{j+1}\right)_{x, i}+r_{i} \Delta_{0 x, i}^{\beta} \stackrel{\star}{y}^{j+1}-d_{i} \stackrel{\star}{y}_{i}^{j+1}, \quad i=1, \ldots, N-1, \\
& \Delta_{0 t_{j+1}}^{\alpha} \stackrel{\star}{y_{0}}=\frac{\bar{a}^{(1)} \stackrel{\star}{y}_{x, 0}-\bar{\lambda}_{-} \stackrel{\star}{y}_{0}}{0.5 h}+\frac{\bar{\mu}_{-}}{0.5 h}, \quad x=0, \\
& \Delta_{0 t_{j+1}}^{\alpha} \stackrel{\star}{y}_{N}=-\frac{\bar{a}^{(N)} \stackrel{\star}{y}_{\bar{x}, N}+\bar{\lambda}_{+} \stackrel{\star}{y}_{N}}{0.5 h}+0.5 h r_{N} \Delta_{0 x_{N-1}}^{\beta} \stackrel{\star}{y}+\frac{\bar{\mu}_{+}}{0.5 h}, \quad x=\ell, \\
& \stackrel{\star}{y}(x, 0)=0,
\end{aligned}
$$

and $\stackrel{\circ}{y}$ is the solution of inhomogeneous equations (8) with homogeneous boundary conditions (11) and inhomogeneous initial conditions (11):

$$
\begin{aligned}
& \Delta_{0 t_{j+1}}^{\alpha} \stackrel{\circ}{i}_{i}=\left(a \stackrel{\circ}{\bar{x}}^{j+1}\right)_{x, i}+r_{i} \Delta_{0 x, i}^{\beta} \dot{i}^{j+1}-d_{i} \stackrel{*}{y}_{i}^{j+1}-d_{i} \dot{y}_{i}^{j+1}+\varphi_{i}^{j+1}, \quad i=1, \ldots, N-1, \\
& \Delta_{0 t_{j+1}}^{\alpha} \stackrel{\circ}{y}_{0}=\frac{\bar{a}^{(1)} \stackrel{\circ}{y}_{x, 0}-\bar{\lambda}_{-} \stackrel{\circ}{y}_{0}}{0.5 h}, \quad x=0
\end{aligned}
$$

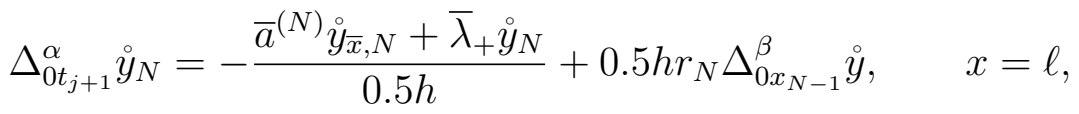

$$
\begin{aligned}
& \stackrel{\circ}{y}(x, 0)=u_{0}(x) .
\end{aligned}
$$

We write problem (13)-16) in the canonical form: at a point $P\left(x_{i}, t_{j+1}\right), i=1, \ldots, N-1$ :

$$
\begin{aligned}
& {\left[\frac{1}{\Gamma(2-\alpha)} \frac{1}{\tau^{\alpha}}+\frac{a_{i+1}+a_{i}}{h^{2}}-\frac{r_{i}}{\Gamma(2-\beta)} \frac{1}{h^{\beta}}+d_{i}\right] \stackrel{\star}{y}_{i}^{j+1}=\frac{a_{i+1}}{h^{2}} \stackrel{\star}{y+1}_{i+1}^{j+1}+\left[\frac{a_{i}}{h^{2}}-\frac{r_{i}\left(2-2^{1-\beta}\right)}{\Gamma(2-\beta) h^{\beta}}\right] \grave{y}_{i-1}^{j+1}} \\
& -\frac{r_{i}}{\Gamma(2-\beta) h} \sum_{s=1}^{i-2}\left(-x_{i-s+1}^{1-\beta}+2 x_{i-s}^{1-\beta}-x_{i-s-1}^{1-\beta}\right) \stackrel{\star}{y}_{s}^{j+1}-\frac{r_{i}}{\Gamma(2-\beta) h}\left(x_{i}^{1-\beta}-x_{i-1}^{1-\beta}\right) \stackrel{\star}{y}_{0}^{j+1} \\
& +\frac{\left(2-2^{1-\alpha}\right)}{\Gamma(2-\alpha) \tau^{\alpha}} \grave{y}_{i}^{j}+\frac{1}{\Gamma(2-\alpha) \tau} \sum_{s=1}^{j-1}\left(-t_{j-s+2}^{1-\alpha}+2 t_{j-s+1}^{1-\alpha}-t_{j-s}^{1-\alpha}\right) \stackrel{y}{y}_{i}^{s}++\frac{1}{\Gamma(2-\alpha) \tau}\left(t_{j+1}^{1-\alpha}-t_{j}^{1-\alpha}\right) \star_{i}^{\star 0} ;
\end{aligned}
$$


at a point $P\left(x_{0}, t_{j+1}\right), j=1,2, \ldots$ :

$$
\begin{aligned}
& {\left[\frac{1}{\Gamma(2-\alpha) \tau^{\alpha}}+\frac{\bar{a}^{(1)}}{0.5 h^{2}}+\frac{\bar{\lambda}_{-}}{0.5 h}\right] \stackrel{\star}{y}_{0}^{j+1}=\frac{\bar{a}^{(1)}}{0.5 h^{2}} \hat{y}_{0}^{j+1}+\frac{2-2^{1-\alpha}}{\Gamma(2-\alpha) \tau^{\alpha}} \hat{y}_{0}^{j}} \\
& +\frac{1}{\Gamma(2-\alpha) \tau} \sum_{s=0}^{j-2}\left(-t_{j-s+1}^{1-\alpha}+2 t_{j-s}^{1-\alpha}-t_{j-s-1}^{1-\alpha}\right) \hat{y}_{0}^{\star s+1}+\frac{1}{\Gamma(2-\alpha) \tau}\left(t_{j+1}^{1-\alpha}-t_{j}^{1-\alpha}\right) \hat{y}_{0}^{\star 0}+\frac{\bar{\mu}_{-}}{0.5 h}
\end{aligned}
$$

at a point $P\left(x_{N}, t_{j+1}\right), j=1,2, \ldots$ :

$$
\begin{aligned}
& {\left[\frac{1}{\Gamma(2-\alpha)} \frac{1}{\tau^{\alpha}}+\frac{\bar{a}^{(N)}}{0.5 h}+\frac{\bar{\lambda}_{+}}{0.5 h}\right] \stackrel{\star}{y}_{N}^{j+1}=\left[\frac{\bar{a}^{(N)}}{0.5 h}+\frac{0.5 h r_{N}\left(2^{1-\beta}-1\right)}{\Gamma(2-\beta) h^{\beta}}\right] \grave{y}_{N-1}^{j+1}} \\
& +\frac{\left(2-2^{1-\alpha}\right)}{\Gamma(2-\alpha) \tau^{\alpha}} \stackrel{\star}{y}_{N}^{j}+\frac{1}{\Gamma(2-\alpha) \tau} \sum_{s=1}^{j-1}\left(-t_{j-s+2}^{1-\alpha}+2 t_{j-s+1}^{1-\alpha}-t_{j-s}^{1-\alpha}\right) \grave{y}_{N}^{\star s}+\frac{1}{\Gamma(2-\alpha) \tau}\left(t_{j+1}^{1-\alpha}-t_{j}^{1-\alpha}\right) \grave{y}_{N}^{\star 0} \\
& -\frac{r_{N}}{\Gamma(2-\beta) h} \sum_{s=1}^{N-2}\left(-x_{i-s+1}^{1-\beta}+2 x_{i-s}^{1-\beta}-x_{i-s-1}^{1-\beta}\right) \stackrel{x}{y}_{s}^{j+1}-\frac{r_{N}}{\Gamma(2-\beta) h}\left(x_{N}^{1-\beta}-x_{N-1}^{1-\beta}\right) \stackrel{y}{y}_{0}^{\star j+1} .
\end{aligned}
$$

Hence, $A(P)>0, B(P, Q)>0, D(P)>0$, and thanks for the maximum principle, for small $h$, the solution $\stackrel{y}{y}$ to problem (13)-(16) satisfies the estimate:

$$
\left\|\stackrel{y}{y}^{j+1}\right\|_{C} \leqslant \frac{1}{\lambda^{*}} \max _{0<t^{\prime} \leqslant j \tau}\left(\left|\bar{\mu}_{-}\left(x, t^{\prime}\right)\right|+\left|\bar{\mu}_{+}\left(x, t^{\prime}\right)\right|\right) .
$$

We proceed to estimating the function $\stackrel{\circ}{y}$. We rewrite equation $(17)$ as

$$
\frac{1}{\Gamma(2-\alpha)} \tau^{1-\alpha} \dot{y}_{t}^{j}=\Lambda y^{j+1}+\widetilde{\varphi}^{j+1}
$$

where

$$
\widetilde{\varphi}^{j+1}=\varphi^{j+1}-\frac{1}{\Gamma(2-\alpha)} \sum_{s=0}^{j-1}\left(t_{j-s+1}^{1-\alpha}-t_{j-s}^{1-\alpha}\right) \stackrel{\varphi}{y}_{t}^{s} .
$$

We reduce equation (21) to the canonical form:

$$
\begin{aligned}
& {\left[\frac{1}{\Gamma(2-\alpha)} \frac{1}{\tau^{\alpha}}+\frac{a_{i+1}+a_{i}}{h^{2}}-\frac{r_{i}}{\Gamma(2-\beta)} \frac{1}{h^{\beta}}+d_{i}\right] \grave{y}_{i}^{j+1}=\frac{a_{i+1} \grave{y}_{i+1}^{j+1}}{h^{2}}+\left[\frac{a_{i}}{h^{2}}-\frac{r_{i}\left(2-2^{1-\beta}\right)}{\Gamma(2-\beta) h^{\beta}}\right] \grave{y}_{i-1}^{j+1}} \\
& -\frac{r_{i}}{\Gamma(2-\beta) h} \sum_{s=1}^{i-2}\left(-x_{i-s+1}^{1-\beta}+2 x_{i-s}^{1-\beta}-x_{i-s-1}^{1-\beta}\right) \grave{y}_{s}^{j+1}-\frac{r_{i}}{\Gamma(2-\beta) h}\left(x_{i}^{1-\beta}-x_{i-1}^{1-\beta}\right) \dot{y}_{0}^{j+1}+\Phi^{j+1} \text {, }
\end{aligned}
$$

where

$$
\begin{aligned}
\Phi^{j+1} & =\frac{\left(2-2^{1-\alpha}\right)}{\Gamma(2-\alpha) \tau^{\alpha}} \stackrel{y}{i}_{i}^{j}+\bar{\varphi}^{j+1}, \\
\bar{\varphi}^{j+1} & =\varphi^{j+1}+\frac{1}{\Gamma(2-\alpha)} \frac{1}{\tau}\left(t_{2}^{1-\alpha}-t_{1}^{1-\alpha}\right) \stackrel{y}{y}_{i}^{j-1}-\frac{1}{\tau} \frac{1}{\Gamma(2-\alpha)} \sum_{s=0}^{j-1}\left(t_{j-s+1}^{1-\alpha}-t_{j-s}^{1-\alpha}\right)\left(\stackrel{\circ}{i}_{i}^{s}-\dot{y}_{i}^{s-1}\right) .
\end{aligned}
$$

Let us check the assumptions of Theorem 4 in [48, Ch. V, App., Sect. 2, Eqs. (25)-(27)]. At a point $P_{i}^{j+1}=P\left(x_{i}, t_{j+1}\right)$ we have

$$
\begin{aligned}
& A\left(P_{i}^{j+1}\right)=\left[\frac{1}{\Gamma(2-\alpha)} \frac{1}{\tau^{\alpha}}+\frac{a_{i+1}+a_{i}}{h^{2}}-\frac{r_{i}}{\Gamma(2-\beta)} \frac{1}{h^{\beta}}+d_{i}\right]>0, \\
& B\left(P_{i}^{j+1}, Q\right)=\left\{\frac{a_{i+1}}{h^{2}} ;\left(\frac{a_{i}}{h^{2}}-\frac{r_{i}\left(2-2^{1-\beta}\right)}{\Gamma(2-\beta) h^{\beta}}\right) ;\right.
\end{aligned}
$$




$$
\begin{aligned}
& -\frac{r_{i}}{\Gamma(2-\beta) h}\left(-x_{i-s+1}^{1-\beta}+2 x_{i-s}^{1-\beta}-x_{i-s-1}^{1-\beta}\right), s=1, \ldots, i-2 ;-\frac{r_{i}}{\Gamma(2-\beta) h}\left(x_{i}^{1-\beta}-x_{i-1}^{1-\beta}\right) \\
& D^{\prime}\left(P_{i}^{j+1}\right)=A\left(P_{i}^{j+1}\right)-\sum_{Q \in \mathbb{I}^{\prime}\left(P_{i}^{j+1}\right)} B\left(P_{i}^{j+1}, Q\right)=\frac{1+\Gamma(2-\alpha) \tau^{\alpha} d_{i}}{\Gamma(2-\alpha) \tau^{\alpha}}>0,
\end{aligned}
$$

for all $Q \in \mathbb{I I}^{\prime \prime}, Q \in \mathbb{I I}^{\prime}$,

$$
\begin{aligned}
& \sum_{Q \in \mathbb{I}^{\prime \prime}} B\left(P_{j+1}, Q\right)=\frac{1}{\Gamma(2-\alpha) \tau^{\alpha}}>0 \\
& \frac{1}{D^{\prime}\left(P_{j+1}\right)} \sum_{Q \in \mathbb{I}_{j}^{\prime \prime}} B\left(P_{j+1}, Q\right)=\frac{1}{1+\Gamma(2-\alpha) \tau^{\alpha} d_{i}} \leqslant 1
\end{aligned}
$$

where

$$
\begin{aligned}
& \mathbb{I}_{\left(P\left(x, t_{j+1}\right)\right)}^{\prime}=\mathbb{I}_{j+1}^{\prime}+\mathbb{I}_{j}^{\prime \prime}, \\
& \mathbb{I}^{\prime} \text { is the set of nodes } Q=Q(\xi, t) \in \mathbb{I}_{\left(P\left(x, t_{j}\right)\right)}^{\prime}, \\
& \mathbb{I}_{j}^{\prime \prime} \text { is the set of nodes } Q=Q\left(\xi, t_{j}\right) \in \mathbb{I}_{\left(P\left(x, t_{j}\right)\right)}^{\prime} .
\end{aligned}
$$

Due to Theorem 4 in [48, Ch. V, App.] and (24) we obtain the estimate:

$$
\left\|\stackrel{\circ}{j+1}^{j+1}\right\|_{C} \leqslant \frac{2-2^{1-\alpha}}{1+\Gamma(2-\alpha) \tau^{\alpha} d_{i}}\left\|\dot{y}^{j}\right\|_{C}+\Gamma(2-\alpha) \tau^{\alpha}\left\|\bar{\varphi}^{j+1}\right\|_{C} .
$$

Let us estimate $\left\|\bar{\varphi}^{j+1}\right\|_{C}$, where

$$
\bar{\varphi}^{j+1}=\varphi^{j+1}+\frac{1}{\Gamma(2-\alpha) \tau} \sum_{s=0}^{j-2}\left(-t_{j-s+1}^{1-\alpha}+2 t_{j-s}^{1-\alpha}-t_{j-s-1}^{1-\alpha}\right) \grave{y}^{s+1}++\frac{1}{\Gamma(2-\alpha) \tau}\left(t_{j+1}^{1-\alpha}-t_{j}^{1-\alpha}\right) \grave{y}^{0} .
$$

By (26) we obtain the estimate

$$
\left\|\bar{\varphi}^{j+1}\right\|_{C} \leqslant\left\|\varphi^{j+1}\right\|_{C}+\frac{2^{1-\alpha}-1}{\tau^{\alpha} \Gamma(2-\alpha)} \max _{0 \leqslant s \leqslant j-2}\left\|\dot{y}^{s}\right\|_{C}
$$

Therefore, for $\stackrel{y}{y}$ the estimate holds:

$$
\left\|\dot{y}^{j+1}\right\|_{C} \leqslant\left\|\dot{y}^{0}\right\|_{C}+\Gamma(2-\alpha) \sum_{j^{\prime}=0}^{j} \tau^{\alpha}\left\|\varphi^{j^{\prime}}\right\|_{C}
$$

Thus, estimates (21) and (28) imply the final estimate (7).

1.4. Fractional order diffusion equation with convection. While increasing the approximation order of Robin boundary condition up to $O\left(h^{2-\beta}+\tau^{2-\alpha}\right)$ we have obtained a difference scheme with a nonlocal in the spatial variable $x$ boundary condition, which is not the case in problem if in problem (2)-(4) equation (2) is replaced by

$$
\partial_{0 t}^{\alpha} u=\frac{\partial}{\partial x}\left(k(x, t) \frac{\partial u}{\partial x}\right)+r(x, t) \frac{\partial u}{\partial x}-q(x, t) u+f(x, t), \quad 0 \leqslant x \leqslant \ell,
$$

where

$$
\begin{aligned}
& 0<c_{1} \leqslant k(x, t) \leqslant c_{2}, \quad 0<q(x, t) \leqslant c_{3}, \quad r(0, t) \geqslant 0, \\
& r(\ell, t) \leqslant 0, \quad|r(x, t)| \leqslant c_{4}, \quad \lambda_{ \pm} \geqslant \lambda^{*}>0,
\end{aligned}
$$

$c_{0}, c_{1}$ are positive constants.

A difference scheme for problem (29), (3), (4) reads as

$$
\Delta_{0 t_{j+1}}^{\alpha} y=\bar{\Lambda} y^{j+1}+\Phi^{j+1}, \quad y(x, 0)=u_{0}(x)
$$


where

$$
\begin{aligned}
& \bar{\Lambda} y=\left\{\begin{array}{ll}
\widetilde{\Lambda} y=\varkappa\left(a y_{\bar{x}}\right)_{x}+b^{+} a^{(+1)} y_{x}+b^{-} a y_{\bar{x}}-d y, & x \in \omega_{h}, \\
\Lambda^{-} y=\frac{\bar{a}^{(1)} y_{x, 0}-\bar{\lambda}_{-} y_{0}}{0.5 h}, & x=0, \\
\Lambda^{+} y=-\frac{\bar{a}^{(N)} y_{\bar{x}, N}+\bar{\lambda}_{+} y_{N}}{0.5 h}, & x=\ell,
\end{array} \quad \Phi= \begin{cases}\varphi, & x \in \omega_{h}, \\
\frac{\bar{\mu}_{-}}{0.5 h}, & x=0, \\
\frac{\bar{\mu}_{+}}{0.5 h}, & x=\ell,\end{cases} \right. \\
& \bar{a}^{(1)}=a^{(1)}+0.5 h r_{0}, \quad \bar{a}^{(N)}=a^{(N)}-0.5 h r_{N}, \quad \bar{\lambda}_{-}=\lambda_{-}+0.5 h d^{(0)}, \\
& \bar{\lambda}_{+}=\lambda_{+}+0.5 h d^{(N)}, \quad \bar{\mu}_{-}=\mu_{-}+0.5 h f_{0}, \quad \bar{\mu}_{+}=\mu_{+}+0.5 h f_{N} .
\end{aligned}
$$

As the solution and data of problem (29), (3), (4) are smooth enough, difference scheme (30) has the approximation order $O\left(h^{2}+\tau^{2-\alpha}\right)$.

\section{Fractional ORDER heAT EQUATION With A CONCENTRATED HEAT CAPACITY}

When a concentrated heat capacity is located on the boundary of a domain, for a heat equation the following boundary condition can be imposed at $x=0$ :

$$
c_{0} \frac{\partial u}{\partial t}=k \frac{\partial u}{\partial x}, \quad c_{0}=\text { const }>0 .
$$

Such conditions arise in the case, when we consider a solid with a high heat capacity [49] and solve a problem on temperature stabilization in a bounded media under the presence of a heater treated as a concentrated heat capacity [50].

Similar problems arise also while controlling a salt regime of soil, when a disintegration of the upper layer is achieved by the water drain from an area flooded for some period [51]. If a water layer of a fixed $h$ is present on the area, on the upper boundary we should impose the condition

$$
h \frac{\partial c}{\partial t}=D \frac{\partial c}{\partial x}
$$

where $c$ is a salt concentration in the soil solution, $D$ is the diffusion coefficient [51].

Before we proceed to formulation of the problem, let us provide an example [28], in which a fractional derivative arises on the boundary without using the fractal conception.

On the half-strip $x>0,0<t<T$, we consider the problem

$$
\begin{aligned}
& u_{t}=\left(k u_{x}\right)_{x}, \\
& k_{1} u_{x}(0, t)=\beta_{1}(t) u(0, t)-\mu_{1}(t), \\
& u(x, 0)=0, \quad|u(x, t)| \leqslant M, \quad 0<x<\infty, \quad 0 \leqslant t \leqslant T, \\
& k(x)= \begin{cases}k_{1}, & x \leqslant x_{1}, \\
k_{2}, & x>x_{1} .\end{cases}
\end{aligned}
$$

At a discontinuity point of $k(x)$, the temperature and the heat flow should be continuous:

$$
[u]_{x=x_{1}}=u\left(x_{1}+0, t\right)-u\left(x_{1}-0, t\right)=0,\left[k \frac{d u}{d x}\right]_{x=x_{1}}=0 .
$$

The solution $u=u^{+}$of problem $(31)-(33)$ in $x_{1}>0, t>0$ is of the form:

$$
u^{+}(x, t)=-\frac{1}{\sqrt{k_{2} \pi}} \int_{0}^{t} \frac{\nu(\tau)}{\sqrt{t-\tau}} \exp \left(-\frac{\left(x-x_{1}\right)^{2}}{4 k_{2}(t-\tau)}\right) d \tau, \quad \nu(t)=k_{2} u_{x}^{+}\left(x_{1}, t\right) .
$$


As $x=x_{1}$ we get:

$$
u^{+}\left(x_{1}+0, t\right)=-\frac{1}{\sqrt{k_{2} \pi}} \int_{0}^{t} \frac{k_{2} u_{x}^{+}\left(x_{1}+0, \tau\right)}{\sqrt{t-\tau}} d \tau .
$$

Due to (34) this implies

$$
u^{-}\left(x_{1}-0, t\right)=-\frac{1}{\sqrt{k_{2} \pi}} \int_{0}^{t} \frac{k_{1} u_{x}^{-}\left(x_{1}-0, \tau\right)}{\sqrt{t-\tau}} d \tau,
$$

where $u^{-}(x, t)$ is a solution of problem (31)-33) in the domain $0<x<x_{1}, 0<t \leqslant T$. Resolving Abel integral equation (35), we obtain

$$
-k_{1} u_{x}^{-}\left(x_{1}-0, \tau\right)=\sqrt{\frac{k_{2}}{\pi}} \frac{d}{d t} \int_{0}^{t} \frac{u^{-}\left(x_{1}, \tau\right)}{(t-\tau)^{\frac{1}{2}}} d \tau .
$$

Thus, while calculating the temperature field in the domain $0<x<x_{1}, t>0$, we can take into consideration the influence of a semi-infinite domain $x>x_{1}, t>0$ by imposing non-local condition $(36)$ at $x=x_{1}$ with a fractional derivative of order $\alpha=\frac{1}{2}$.

We proceed to considering the difference scheme for a fractional order differential equation in the case, when on the boundary a fractional order condition with a concentrated heat capacity is imposed:

$$
c_{0} \partial_{o t}^{\alpha} u=k \frac{\partial u}{\partial x}, \quad c_{0}=\text { const }>0
$$

where $\partial_{o t}^{\alpha} u$ is the Caputo fractional derivative of order $\alpha, 0<\alpha<1$.

2.1. Formulation of problem. In the rectangle $Q_{T}=\{0 \leqslant x \leqslant \ell, 0<t \leqslant T\}$ we consider the initial-boundary value problem

$$
\begin{aligned}
& \partial_{0 t}^{\alpha} u=\frac{\partial}{\partial x}\left(k(x, t) \frac{\partial u}{\partial x}\right)+f(x, t),(x, t) \in Q_{T}, \\
& \left\{\begin{array}{r}
k(x, t) \frac{\partial u}{\partial x}=\varkappa_{-} \partial_{0 t}^{\alpha} u+\beta_{-}(x, t) u-\mu_{-}(x, t), \quad x=0, \\
-k(x, t) \frac{\partial u}{\partial x}=\varkappa_{+} \partial_{0 t}^{\alpha} u+\beta_{+}(x, t) u-\mu_{+}(x, t), \quad x=\ell,
\end{array}\right. \\
& u(x, 0)=u_{0}(x)
\end{aligned}
$$

where

$$
0<c_{0} \leqslant k \leqslant c_{1}, \quad \beta_{ \pm \alpha} \geqslant \beta_{*}>0, \quad \varkappa_{ \pm}=\text {const }>0
$$

and

$$
\partial_{0 t}^{\alpha} u=\frac{1}{\Gamma(1-\alpha)} \int_{0}^{t} \frac{\dot{u}(x, \eta)}{(t-\eta)^{\alpha}} d \eta, \quad \dot{u}=\frac{\partial u}{\partial t},
$$

is the Caputo fractional derivative of order $\alpha, 0<\alpha<1$.

2.2. Difference scheme. We choose an uniform spatial grid with the step $h=\frac{\ell}{N}$, $\bar{\omega}_{h}=$ $\left\{x^{i}=i h: i=0,1, \ldots, N\right\}$. On the segment $[0, T]$ we choose an uniform grid

$$
\bar{\omega}_{\tau}^{\prime}=\left\{0, t_{j}=j \tau, \quad j=0,1, \ldots, j_{0}\right\},
$$

$\omega_{\tau}^{\prime}$ is the set of the nodes of the grid $\bar{\omega}_{\tau}^{\prime}$ obeying $t>0$. 
Employing discrete analogue (1) of a regularized Caputo fractional derivative of order $\alpha$, $0<\alpha<1$, we approximate equation (37) by the implicit scheme. Then we obtain the difference equation:

$$
\frac{1}{\Gamma(2-\alpha)} \sum_{s=0}^{j}\left(t_{j-s+1}^{1-\alpha}-t_{j-s}^{1-\alpha}\right) y_{t}^{s}=\Lambda y^{j+1}+\varphi^{j+1}, \quad x \in \omega_{h}
$$

where

$$
\Lambda y=\left(a y_{\bar{x}}\right)_{x}, y_{t}^{s}=\frac{y^{s+1}-y^{s}}{\tau} .
$$

We also need to compete equation (40) by boundary and initial conditions; this will be done later.

Let us write a difference analogue for boundary conditions (38):

$$
\left\{\begin{array}{c}
a^{(1)} y_{x, 0}^{j+1}=\varkappa_{-} \Delta_{0 t_{j+1}}^{\alpha} y_{0}^{j+1}+\beta_{-}(x, t) y_{0}^{j+1}-\mu_{-}(x, t), \\
-a^{(N)} y_{\bar{x}, N}^{j+1}=\varkappa_{+} \Delta_{0 t_{j+1}}^{\alpha} y_{0}^{j+1}+\beta_{+}(x, t) y_{0}^{j+1}-\mu_{+}(x, t), \quad x=0,
\end{array} \quad x=0,\right.
$$

Conditions (41) have approximation order $O(h)$. We increase the approximation order up to $O\left(h^{2}+\tau^{2-\alpha}\right)$ on solutions of equation (37). Since

$$
k \frac{\partial u}{\partial x}=a^{(1)} u_{x, 0}^{j+1}-0.5 h\left(\partial_{0 t}^{\alpha} u-f^{j+1}\right)_{0}+O\left(h^{2}\right),
$$

then

$$
a^{(1)} u_{x, 0}^{j+1}-0.5 h\left(\Delta_{0 t_{j+1}}^{\alpha} u-f^{j+1}\right)_{0}=\varkappa_{-} \Delta_{0 t_{j+1}}^{\alpha} u_{0}+\beta_{-} u_{0}^{j+1}-\mu_{-}+O\left(h^{2}\right)+O\left(h \tau^{2-\alpha}\right) .
$$

Neglecting in the above identity the terms of order $O\left(h^{2}\right)$ and $O\left(h \tau^{2-\alpha}\right)$ and replacing $u$ by $y$, we obtain:

$$
\Delta_{0 t_{j+1}}^{\alpha} y=\frac{a^{(1)} y_{x, 0}^{j+1}-\beta_{-} y_{0}^{j+1}}{\varkappa_{-}+0.5 h}+\widetilde{\mu}_{-}, \quad x=0, \quad \widetilde{\mu}_{-}=\frac{\bar{\mu}_{-}}{\varkappa_{-}+0.5 h}, \quad \bar{\mu}_{-}=\mu_{-}+0.5 h f_{0} .
$$

In the same way, as $x=\ell$, we get:

$$
\Delta_{0 t_{j+1}}^{\alpha} y=-\frac{a^{(N)} y_{\bar{x}, N}^{j+1}+\beta_{+} y_{N}^{j+1}}{\varkappa_{+}+0.5 h}+\widetilde{\mu}_{+}, \quad x=\ell, \quad \widetilde{\mu}_{+}=\frac{\bar{\mu}_{+}}{\varkappa_{+}+0.5 h}, \quad \bar{\mu}_{+}=\mu_{+}+0.5 h f_{N} .
$$

Thus, the difference analogue of problem $37-39$ reads as

$$
\Delta_{0 t_{j+1}}^{\alpha} y=\bar{\Lambda} y^{j+1}+\Phi^{j+1}, \quad y(x, 0)=u_{0}(x)
$$

where

$$
\begin{aligned}
& \bar{\Lambda} y=\left\{\begin{array}{ll}
\Lambda y=\left(a y_{\bar{x}}\right)_{x}, & x \in \omega_{h}, \\
\Lambda^{-} y=\frac{a^{(1)} y_{x, 0}^{j+1}-\beta_{-} y_{0}}{\varkappa_{-}+0.5 h}, & x=0, \\
\Lambda^{+} y^{j+1}=-\frac{a^{(N)} y_{\bar{x}, N}+\beta_{+} y_{N}}{\varkappa_{+}+0.5 h}, & x=\ell,
\end{array} \quad \Phi= \begin{cases}\varphi, & x \in \omega_{h}, \\
\widetilde{\mu}_{-}, & x=0, \\
\widetilde{\mu}_{+}, & x=\ell,\end{cases} \right. \\
& \widetilde{\mu}_{-}=\frac{\bar{\mu}_{-}}{\varkappa_{-}+0.5 h}, \quad \bar{\mu}_{-}=\mu_{-}+0.5 h f_{0}, \quad \widetilde{\mu}_{+}=\frac{\bar{\mu}_{+}}{\varkappa_{+}+0.5 h}, \quad \bar{\mu}_{+}=\mu_{+}+0.5 h f_{\alpha, N} \text {. }
\end{aligned}
$$


2.3. Stability and uniform convergence of difference scheme. The following theorem holds true.

Theorem 2. Difference scheme (43) is stable with respect to the initial data and right hand side and the solution of problem (43) satisfies the estimate

$$
\left\|y^{j+1}\right\|_{C} \leqslant\left\|y^{0}\right\|_{C}+\max _{0<t^{\prime} \leqslant j \tau} \frac{1}{\beta_{*}}\left(\left|\bar{\mu}_{-}\left(x, t^{\prime}\right)\right|+\left|\bar{\mu}_{+}\left(x, t^{\prime}\right)\right|\right)+\Gamma(2-\alpha) \sum_{j^{\prime}=0}^{j} \tau^{\alpha}\left\|\varphi^{j^{\prime}}\right\|_{C},
$$

which implies the convergence of scheme (43) in the uniform metric with the rate $O\left(h^{2}+\tau^{2-\alpha}\right)$.

Proof. We again employ the maximum principle for the solution of a general grid equation. We reduce difference equation and boundary conditions (43) to the canonical form:

$$
\begin{aligned}
& {\left[\frac{1}{\Gamma(2-\alpha)} \frac{1}{\tau^{\alpha}}+\frac{a_{i+1}+a_{i}}{h^{2}}\right] y_{i}^{j+1}=\frac{a_{i+1}}{h^{2}} y_{i+1}^{j+1}+\frac{a_{i}}{h^{2}} y_{i-1}^{j+1}+\frac{2-2^{1-\alpha}}{\Gamma(2-\alpha) \tau^{\alpha}} y_{i}^{j}} \\
& +\frac{1}{\tau} \frac{1}{\Gamma(2-\alpha)}\left[\left(t_{j+1}^{1-\alpha}-t_{j}^{1-\alpha}\right) y_{i}^{0}+\left(-t_{j+1}^{1-\alpha}+2 t_{j}^{1-\alpha}-t_{j-1}^{1-\alpha}\right) y_{i}^{1}\right. \\
& \left.+\ldots+\left(-t_{3}^{1-\alpha}+2 t_{2}^{1-\alpha}-t_{1}^{1-\alpha}\right) y_{i}^{j-1}\right]+\varphi^{j+1}, \\
& {\left[\frac{1}{\Gamma(2-\alpha)} \frac{1}{\tau^{\alpha}}+\frac{a_{1}}{\left(0.5 h+\varkappa_{-}\right) h}+\frac{\beta_{-}}{0.5 h+\varkappa_{-}}\right] y_{0}^{j+1}=\frac{a_{1}}{\left(0.5 h+\varkappa_{-}\right) h} y_{1}^{j+1}+\frac{\left(2-2^{1-\alpha}\right)}{\Gamma(2-\alpha) \tau^{\alpha}} y_{0}^{j}} \\
& +\frac{1}{\tau} \frac{1}{\Gamma(2-\alpha)}\left[\left(t_{j+1}^{1-\alpha}-t_{j}^{1-\alpha}\right) y_{0}^{0}+\left(-t_{j+1}^{1-\alpha}+2 t_{j}^{1-\alpha}-t_{j-1}^{1-\alpha}\right) y_{0}^{1}\right. \\
& \left.+\ldots+\left(-t_{3}^{1-\alpha}+2 t_{2}^{1-\alpha}-t_{1}^{1-\alpha}\right) y_{0}^{j-1}\right]+\bar{\mu}_{-} .
\end{aligned}
$$

This implies

$$
D\left(P\left(x, t_{j+1}\right)\right)=0, \quad D\left(P\left(0, t_{j+1}\right)\right)=\frac{\beta_{-}}{0.5 h+\varkappa_{-}} .
$$

In the same way, as $x=\ell$, we get

$$
D\left(P\left(\ell, t_{j+1}\right)\right)=\frac{\beta_{+}}{0.5 h+\varkappa_{-}} .
$$

To estimate the solution of problem (43), we again represent the solution $y$ as the sum

$$
y=\stackrel{\star}{y}+\stackrel{\circ}{y},
$$

where $\stackrel{y}{y}$ is the solution of the problem as $\bar{\mu}_{-}=\bar{\mu}_{+}=0$, and $\stackrel{\star}{y}$ solves the problem as $u_{0}(x)=0$, $\varphi(x, t)=0$. By Theorem 3 in [48], for $\stackrel{*}{y}$ we obtain the estimate:

$$
\left\|\hat{y}^{\star j+1}\right\|_{C} \leqslant \max _{0<t^{\prime} \leqslant j \tau} \frac{1}{\beta_{*}}\left(\left|\bar{\mu}_{-}\left(x, t^{\prime}\right)\right|+\left|\bar{\mu}_{+}\left(x, t^{\prime}\right)\right|\right) .
$$

Since $D\left(P\left(x, t_{j+1}\right)\right)=0$, to estimate $\stackrel{\circ}{y}$, we rewrite equation 45 as

$$
\left[\frac{1}{\Gamma(2-\alpha)} \frac{1}{\tau^{\alpha}}+\frac{a_{i+1}+a^{i}}{h^{2}}\right] \stackrel{\circ}{y}_{i}^{j+1}=\frac{a_{i+1}}{h^{2}} \stackrel{y}{y}_{i+1}^{j+1}+\frac{a_{i}}{h^{2}} y_{i-1}^{j+1}+\frac{\left(2-2^{1-\alpha}\right)}{\Gamma(2-\alpha) \tau^{\alpha}} \grave{y}_{i}^{j}+\Phi\left(P_{j+1}\right),
$$

where

$$
\Phi(P)=\varphi^{j+1}+\frac{1}{\tau} \frac{1}{\Gamma(2-\alpha)}\left[\left(t_{j+1}^{1-\alpha}-t_{j}^{1-\alpha}\right) \grave{y}_{i}^{0}+\left(-t_{j+1}^{1-\alpha}+2 t_{j}^{1-\alpha}-t_{j-1}^{1-\alpha}\right) \grave{y}_{i}^{1}+\ldots\right.
$$




$$
\left.+\left(-t_{3}^{1-\alpha}+2 t_{2}^{1-\alpha}-t_{1}^{1-\alpha}\right) \dot{y}_{i}^{j-1}\right] .
$$

Let us check the assumptions of Theorem 4 in [48]:

$$
D^{\prime}(P)=\frac{1}{\Gamma(2-\alpha) \tau^{\alpha}}>0, \quad P=P\left(x, t_{j+1}\right), \quad A(P)>0, \quad B((P), Q)>0
$$

for all $Q \in \mathbb{I I I}_{j}^{\prime \prime}, Q \in \mathbb{I I}^{\prime}$, and

$$
\sum_{Q \in \mathbb{I}_{j}^{\prime \prime}} B(P, Q)=\frac{2-2^{1-\alpha}}{\Gamma(2-\alpha) \tau^{\alpha}}>0, \quad \frac{1}{D^{\prime}(P)} \sum_{Q \in \mathbb{I}_{j}^{\prime \prime}} B(P, Q)=2-2^{1-\alpha} \leqslant 1 .
$$

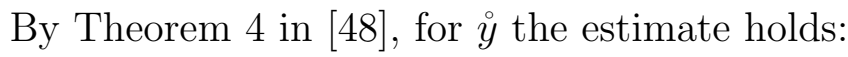

$$
\left\|\dot{y}^{j+1}\right\|_{C} \leqslant\left\|\dot{y}^{0}\right\|_{C}+\Gamma(2-\alpha) \sum_{j^{\prime}=0}^{j} \tau^{\alpha}\left\|\varphi^{j^{\prime}}\right\|_{C} .
$$

Estimates 46) and 47) imply final estimate (44).

Remark 1. If instead of implicit scheme we consider a more general difference equation with weights $\Lambda\left(\sigma y^{j+1}+(1-\sigma) y^{j}\right)$ in the right hand side (40), then a condition for the step $\tau$ arises:

$$
\tau \leqslant \frac{\left(2-2^{1-\nu}\right) h^{2}}{2 c_{1} \Gamma(2-\nu)(1-\sigma)}, \quad 0 \leqslant \sigma \leqslant 1,
$$

which as $\nu \rightarrow 1$, becomes the known condition

$$
\tau \leqslant \frac{h^{2}}{2 c_{1}(1-\sigma)}, \quad 0 \leqslant \sigma \leqslant 1 .
$$

Similar restrictions for the step $\tau$ arise in other problems considered in the paper.

\section{BIBLIOGRAPHY}

1. V.E. Tarasov. Models in theoretical physics with integro-differentiation of fractional order Izhevskij Inst. Komp. Issl. Izhevsk (2011). (in Russian).

2. V.Kh. Shogenov, A.A. Akhkubekov, R.A. Akhkubekov. Method of fracional differentiation in theory of Brownian motion // Izv. VUZov. Sever.-Kavkaz. Region. 1, 46-49 (2004). (in Russian).

3. F. Mainardi, Y. Luchko, G. Pagnini. The fundamental solution of the space-time fractional diffusion equation // Fract. Calc. Appl. Anal. 4:2, 153-192 (2002).

4. E. Scalas, R. Gorenflo, F. Mainardi. Uncoupled continuous-time random walks: solution and limiting behaviour of the master equation // Phys. Rev. E. 69:1, id 011107 (2004).

5. Y. Zhang, D.A. Benson, M.M. Meerschaert, H.P. Scheffler. On using random walks to solve the space fractional advectional-dispersion equations // J. Stat. Phys. 123:1, 89-110 (2006).

6. N.G. Abrashina-Zhadaeva, I.A. Timoshchenko. Finite-difference schemes for a diffusion equation with fractional derivatives in a multidimensional domain // Differ. Uravn. 49:7, 819-825 (2013). [Diff. Equat. 49:7, 789-795 (2013).]

7. O.Yu. Dinariev. Flow in a fractured medium with fractal fracture geometry // Izv. Akad. Nauk SSSR. Mekh. Zhidk. Gaza. 5, 66-70 (1990). [Fluid Dyn. 25:5, 704-708 (1990).]

8. V.L. Kobelev, Ya.L. Kobelev, E.P. Romanov Non-Debye relaxation and diffusion in a fractal space // Dokl. RAN. 361:6, 755-758 (1998). [Dokl. Phys. 43:8, 487-490 (1998).]

9. Ya.L. Kobelev, L.Ya. Kobelev, E.P. Romanov. Self-maintained processes in the case of nonlinear fractal diffusion // Dokl. Akad. Nauk. 369:3, 332-333 (1999). [Dokl. Phys. 44:11, 752-753 (1999).]

10. A.N. Kochubei. Fractional-order diffusion // Differ. Uravn. 26:4, 660-670 (1990). [Differ. Equat. 26:4, 485-492 (1990).]

11. V.Kh. Shogenov, S.K. Kumykova, M. Kh. Shkhanukov-Lafishev // Dokl. Adyg. Mezhd. Akad. Nauk. 2:1, 43-45 (1996). (in Russian). 
12. G.I. Barenblatt, Yu.P. Zheltov. Fundamental equations of filtration of homogeneous liquids in fissured rocks // Dokl. Akad. Nauk. 132:3, 545-548 (1960). [Sov. Phys. Dokl. 5, 522-525 (1960).]

13. R.R. Nigmatullin. The realization of generalized transfer equation in a medium with fractal geometry // Phys. Status Solidi. B. 133:1, 425-430 (1986).

14. K.V. Chukbar. Stochastic transport and fractional derivatives // Zhur. Exper. Teor. Fiz. 108:5(11), 1875-1884 (1995). [J. Exp. Theor. Phys. 81:5, 1025-1029 (1995).]

15. V.M. Goloviznin, I.A. Korotkin. Numerical methods for some one-dimensional equations with fractional derivatives // Differ. Uravn. 42:7, 907-913 (2006). [Diff. Equat. 42:7, 967-973 (2006).]

16. R.R. Nigmatullin. Relaxation features of systems with residual memory // Phys. Solid State. 27:5, 1583-1585 (1985). (in Russian).

17. V.M. Goloviznin, V.P. Kiselev. I.A. Korotkin. Numerical methods of solving diffusion equation with a fractional derivative in one-dimensional case // Preprint IBRAE-2003-12, IBRAE RAN, Moscwo (2003). (in Russian).

18. V.M. Goloviznin, V.P. Kiselev. I.A. Korotkin, Yu.P. Yurkov. Direct problems of classical transfer of radionuclides in geological formations // Izv. RAN. Energ. 4, 121-130 (2004). (in Russian).

19. A.M. Nakhushev. Fractional calculus and its application. Fizmatgiz, Moscow (2003). (in Russian).

20. V.V. Uchaikin. Anomalous diffusion of particles with a finite free-motion velocity // Teor. Matem. Fiz. 115:1, 154-160. [Theor. Math. Phys. 115:1, (1998).]

21. V.Yu. Zaburdaev, K.V. Chukbar. Enhanced superdiffusion and finite velocity of Levy flights // Zhurn. Exper. Teor. Fiz. 121:2, 299-307 (2002). [J. Exp. Theor. Phys. 94:2, 252-259 (2002).]

22. J. Klafter, M.F. Shlesinger, G. Zumofen. Beyond Brownian motion // Phys. Today. 49:2, 33-339 (1996).

23. Bangti Jin, Raytcho Lazarov, Zhi Zhou. A Petrov-Galerkin finite element method for fractional convection-diffusion equations // SIAM J. Nummer. Anal. 54:1, 481-503 (2014).

24. Bangti Jin, Raytcho Lazarov, Josef Pasciak, Zhi Zhou. Error analysis of a finite element method for the space-fractional parabolic equation // SIAM J. Nummer. Anal. 52:5, 2272-2294 (2016).

25. B. Jin, R. Lazarov, Z. Zhou. An analysis of the $L_{1}$ scheme for the subdiffusion equation with nonsmooth data // IMA J. Numer. Anal. 36:1, 197-221 (2016).

26. M.M. Lafisheva, M.Kh. Shkhanukov-Lafishev. Locally one-dimensional difference schemes for the fractional order diffusion equation // Zhur. Vychisl. Matem. Matem. Fiz. 48:10, 1878-1887 (2008). [Comp. Math. Math. Phys. 48:10, 1875-1884 (2008).]

27. A.K. Bazzaev, M.Kh. Shkhanukov-Lafishev. Locally-one-dimensional scheme for fractional diffusion equation with Robin boundary conditions // Zhur. Vychisl. Matem. Matem. Fiz. 50:7, 12001208 (2010). [Comp. Math. Math. Phys. 50:7, 1141-1149 (2010).]

28. A.K. Bazzaev, A.V. Mambetova, M.Kh. Shkhanukov-Lafishev. Locally one-dimensional scheme for the heat equation of fractional order with concentrated heat capacity // Zhurn. Vychisl. Matem. Matem. Fiz. 52:9, 1656-1665 (2012). (in Russian).

29. A.K. Bazzaev. Finite-difference schemes for diffusion equation of fractional order with third type boundary conditions in multidimensional domain // Ufimskij Matem. Zhurn. 5:1, 11-16 (2013). [Ufa Math. J. 5:1, 11-16 (2013).]

30. A.K. Bazzaev, M.Kh. Shkhanukov-Lafishev. Locally one-dimensional schemes for the diffusion equation with a fractional time derivative in an arbitrary domain // Zhurn. Vychisl. Matem. Matem. Fiz. 56:1, 113-123 (2016). [Comput. Math. Math. Phys. 56:1, 106-115 (2016).]

31. A.A. Alikhanov. Boundary value problems for the diffusion equation of the variable order in differential and difference settings // Appl. Math. Comput. 219:8, 3938-3946 (2012).

32. K. Diethelm, G. Walz. Numerical solution of fractional order differential equations by extrapolation // Numer. Algorithms. 16:3-4, 231-253 (1997).

33. K. Diethelm, N.J. Ford. Analysis of fractional differential equations // J. Math. Anal. Appl. 265:2, 229-248 (2002).

34. Yu. Povstenko. Axisymmetric solutions to fractional diffusion-wave equation in a cylinder under Robin boundary condition // Eur. Phys. J.-Spec. 222:8, 1767-1777 (2013).

35. Yu. Povstenko. Time-fractional heat conduction in an infinite medium with a spherical hole under Robin boundary condition // Fract. Calc. Appl. Anal. 16:2, 354-369 (2013). 
36. Ch. Tadjeran, M. Meerschaert, H. Scheffler. A second-order accurate numerical approximation for the fractional diffusion equation // J. Comp. Phys. 213:1, 205-213 (2006).

37. Yu. Luchko. Maximum principle for the generalized time-fractional diffusion equation // J. Math. Anal. Appl. 351:1, 218-223 (2009).

38. Yu. Luchko. Boundary value problems for the generalized time-fractional diffusion equation of distributed order // Fract. Calc. Appl. Anal. 12:4, 409-422 (2009).

39. Yu. Luchko. Maximum principle and its application for the time-fractional diffusion equations // Fract. Calc. Appl. Anal. 14:1, 110-124 (2011).

40. Yu. Luchko. Some uniqueness and existence results for the initial-boundary-value problems for the generalized time-fractional diffusion equation // Comp. Math. Appl. 59:5, 1766-1772 (2010).

41. Juan J. Nieto. Maximum principles for fractional differential equations derived from Mittag-Leffler functions // Appl. Math. Lett. 23:10, 1248-1251 (2010).

42. H. Ye, F. Liu, V. Anh, I. Turner. Maximum principle and numerical method for the multi-term time-space Riesz-Caputo fractional differential equations // Appl. Math. Comp. 227, 531-540 (2014).

43. A. K. Bazzaev, M. Kh. Shhanukov-Lafishev. On the convergence of difference schemes for fractional differential equations with Robin boundary conditions // Zhurn. Vychisl. Matem. Matem. Fiz. 57:1, 122-132 (2017). [Comp. Math. Math. Phys. 57:1, 133-144 (2017).]

44. F.I. Taukenova, M.Kh. Shkhanukov-Lafishev. Difference methods for solving boundary value problems for fractional differential equations // Zhur. Vychisl. Matem. Matem. Fiz. 46:10, 1871-1881 (2006). [Comp. Math. Math. Phys. 46:10, 1785-1795 (2006).]

45. A.A. Alikhanov. Stability and convergence of difference schemes for boundary value problems for the fractional-order diffusion equation // Zhurn. Vychisl. Matem. Matem. Fiz. 56:4, 572-586 (2016). [Comp. Math. Math. Phys. 56:4, 561-575 (2016).]

46. A.K. Bazzaev. Robin boundary value problem for a generalized parabolic equations with a fractional derivative in time in a multi-dimensional domain // Vestnik VGU. Ser. Fiz. Matem. 2, 5-14 (2010). (in Russian).

47. A.A. Samarski. The theory of difference schemes. Nauka, Moscow, 1989. [Marcel Dekker, New York (2001).]

48. A.A. Samarskii, A.V. Gulin. Stability of finite-difference schemes. Nauka, Moscow. 1973. (in Russian).

49. A.N. Tikhonov, A.A. Samarskii. Equations of mathematical physics. Nauka, Moscow (1996). [Int. Ser. Monog. Pure Appl. Math. 39. Pergamon Press, Oxford (1963).]

50. A.A. Samarskii. On one boundary value problem on heat conduction // in "Izbran. tr. A.A. Samarskogo". Maks Press, Moscow, 1-22 (2003). (in Russian).

51. S.V. Nerpin, A.F. Chudnovsky. Energy- and mass-transfer in a system plant-soil-air. Gidrometeoizdat, Leningrad (1975). (in Russian).

Alexander Kazbekovich Bazzaev,

Khetagurov North-Ossetia State University,

Vatutina str., 44-46,

362025, Vladikavkaz, Russia

Vladikavkaz Administration Institute,

Borodinskaya str., 14,

362025, Vladikavkaz, Russia

E-mail: albazzaev@gmail.ru

Igor Dzastemirovich Tsopanov,

Vladikavkaz Administration Institute,

Borodinskaya str., 14,

362025, Vladikavkaz, Russia

E-mail: 55tsopanovig@gmail.com 